\title{
Clínica de la histeria masculina
}

\section{Clinic of male hysteria}

\section{Luciano Lutereau}

\section{RESUMEN:}

Este artículo se propone interrogar la histeria en el varón, a partir de la pregunta relativa a la incidencia que la especificidad masculina presta al tipo clínico, en la medida en que, para aquella, la vía fálica requiere una puesta a prueba de la potencia y la posibilidad de su sintomatización. Así es que puede advertirse la importancia de distinguir entre histeria masculina, posición seductora y homosexualidad, para que se plantee la verdadera diferencia clínica significativa: aquella por la cual hoy en día muchos casos de histeria masculina se confunden con la neurosis obsesiva, o con cuadros como la fobia o borderlines.

Palabras clave:

Psicoanálisis - Lacan - Neurosis -

Histeria - Masculinidad

\section{SumMARY:}

This article aims to interrogate the hysteria in the male, from the question concerning the incidence that the masculine specificity lends to the clinical type, considering that, for that, the phallic way requires a test of the power and the possibility of its symptomization. Thus, the importance of distinguishing between male hysteria, seductive position, and homosexuality can be seen in order to raise the true significant clinical difference: that for which today many cases of male hysteria are confused with obsessive neurosis, or with pictures as phobia or borderlines.

\section{WORD PAD:}

Psychoanalysis - Lacan - Neurosis Hysteria - Masculinity 



\section{ClíniCa DE LA HISTERIA MASCULINA}

La histeria masculina es un problema clínico para el psicoanálisis. No sólo por la poca cantidad de casos que suelen publicarse, sino por un motivo conceptual: la histeria, cuyo fundamento es la identificación y la búsqueda de un ser (asociado al deseo, que siempre es deseo del Otro) hunde sus raíces en la feminidad o, mejor dicho, en la posición subjetiva de la mujer, para quien la falta es un aspecto de estructura.

Mujer no es el ser hablante que tiene un órgano anatómico, u otro, sino quien se sitúa respecto del significante que distribuye el sexo (el falo) en la vía del ser. Mujer es el ser hablante que se posiciona en relación a la demanda amorosa, para obtener un ser (ser amada) y, en todo caso, por este motivo la histeria es fundamentalmente femenina, ya que este tipo clínico intercambia un modo de la pregunta por el ser, por otro modo (ser deseada). La histeria femenina interroga el deseo del Otro (con el cual se identifica) para ubicar el objeto que le corresponde, con el que se ubica en relación de exclusión. Esta estructura de tres términos (el Otro, el objeto, la histérica) es lo que establece la pregnancia de "la Otra" en la histeria femenina.

Ahora bien, cuando interrogamos la histeria masculina surgen algunas preguntas: por un lado, si la posición de "ser el falo" es femenina, cabría reconocer que también al varón le toca una parte de esta feminización, no sólo en su infancia (en relación al amor de la madre) sino también en la actitud que muchos varones pueden adoptar en la vida adulta cuando han rechazado simbolizar la potencia fálica por la vía del tener. En este sentido es que hoy en día se habla de hombres "feminizados" (o “histerizados”) para nombrar las más diversas conductas de seducción en que un varón puede quedar fijado.

Por otro lado, la histeria masculina plantea otra pregunta respecto de su diferencia con la homosexualidad masculina: si esta última —en términos generalesimplica una posición narcisista (en la que retorna la cuestión del ser), ¿cómo distinguir entre el homosexual y el histérico? En este punto, lo que esta pregunta - tanto como la anterior- demuestra es un problema del psicoanálisis que aquí no abordaremos, pero cuyo nudo hace posible ubicar el surgimiento de la necesidad de estas distinciones: la confusión entre posición pasiva, homosexual y feminización. Quien trabajó esta cuestión es Silvia Bleichmar (2006), quien destacara el modo en que habitualmente se pudo confundir la histeria masculina con una homosexualidad reprimida (o latente), sin apreciar incluso que eventualmente la posición pasiva ante el padre es constitutiva de la masculinidad.

He aquí, entonces, el problema del Edipo negativo en el varón, cuyo alcance ha sido poco explorado y que, en el caso de la histeria masculina, lleva al prejuicio de suponer que es una posición simétrica a la de la histeria femenina; de ahí que muchas veces se la intente diagnosticar a través del lugar de la Otra (o el goce que se le supone a la mujer), vía fantasmática que renuncia a que sea el mecanismo de formación de síntoma lo que determine el tipo clínico $y$, por ejemplo, que indetermina especialmente el diagnóstico (ya que incluso en un psicótico como Schreber encontramos fantasías histéricas y, por cierto, ¿cómo distinguir entre una fantasía histérica en un psicótico y el empuje a la mujer?).

Este es el punto más complejo para interrogar la histeria en el varón, la pregunta a propósito de la incidencia que la especifi- 
cidad masculina presta al tipo clínico, en la medida en que, para aquella, la vía fálica requiere una puesta a prueba de la potencia y la posibilidad de su sintomatización. Dicho de otra manera, ¿qué fin presta la histeria al varón para sintomatizar su potencia? Así es que puede advertirse la importancia de distinguir entre histeria masculina, posición seductora y homosexualidad, para que se plantee la verdadera diferencia clínica significativa: aquella por la cual hoy en día muchos casos de histeria masculina se confunden con la neurosis obsesiva (tan solo porque se trata de una sintomatización de la potencia), o con cuadros como la fobia o borderlines (por la presencia masiva de angustia).

El primer eje clínico que trazaremos podría ser graficado en función de los tiempos del Edipo: posición seductora (primer tiempo), homosexualidad (segundo tiempo), histeria (tercer tiempo), donde la distinción clínica es crucial para no diagnosticar como neurosis posiciones que no son neuróticas. Sólo en respuesta a la encrucijada que plantea el tercer tiempo del Edipo -y que para el varón implica situarse respecto de la potencia del padre, como seducido antes que como seductor- es que puede hablarse de una neurosis stricto sen$s u$. Este es uno de los grandes problemas del psicoanálisis de nuestro tiempo, la utilización de los tipos clínicos más allá de su razón pertinente, que desdibuja el segundo eje clínico que tomaremos: la distinción entre histeria masculina y otras formas de neurosis, donde el problema concreto que encontramos es el siguiente: hoy en día se diagnostica como histeria masculina casos que no son de neurosis, y los casos de histeria masculina quedan encubiertos bajo otras formas de neurosis.

\section{¿Histeria, SEDUCCIÓN O VARONES FEMINIZADOS?}

En el año 2005, el músico Gabo Ferro editó el disco Canciones que un hombre no debería cantar. El título proviene de la frase que, en 1959, Édith Piaf habría dicho luego de escuchar a Jacques Brel cantar Ne me quitte pas: “¡Un hombre no debería cantar cosas así!”, exclamó el gorrión de París.

En dicha canción, Brel interpretaba a un hombre que suplicaba no ser abandonado. ¿Qué puede tener de escandaloso un gesto semejante? En palabras de Ferro: “¿Acaso ver a un hombre en el lugar que cierta (gran) parte de la sociedad y la cultura venían (con pocas excepciones) colocando a la mujer?". Asimismo, acompaña esta pregunta con otra: “¿Qué cosas deberíamos, entonces, cantar los hombres?”.

Desde hace algunos años se habla, en el contexto del psicoanálisis, de cierta "feminización del mundo". La expresión es curiosa: retoma, por un lado, la llamada “estetización de la vida cotidiana”, de la que algunos filósofos han hablado desde los '80 hasta nuestros días; pero también, por otro, agrega un matiz suplementario, referido a una cuestión de las posiciones sexuadas.

En sentido amplio, la concepción vulgar entiende esta expresión en función de una mayor disposición de las mujeres para acceder a lugares anteriormente ocupados por varones. No obstante, no podría afirmarse con certeza que esto sea algo universal, como tampoco que este acceso sea un índice de feminidad. En varios casos no demuestra más que la aptitud masculina de algunas mujeres, su competencia para la destreza fálica. 
Estas líneas avanzan en sentido contrario. Antes que un ascenso de lo femenino a la esfera pública, determinados fenómenos sociales contemporáneos demuestran que los hombres (varones y mujeres) ya no tienen interés en continuar asociados a la potencia del falo. Esta podría ser una forma menos tonta de entender el desenlace del patriarcado: ya no hay hombres... en el sentido tradicional de la palabra.

Pensemos un ejemplo. Suele hablarse hoy en día de "femicidios". Evaluar la pertinencia de esta categoría es poco interesante. Mejor atendamos a la circunstancia siguiente: se vincularía este tipo de violencia con la consideración de la mujer como objeto. Sin embargo, en diferentes casos se comprueba todo lo contrario; es lo que ocurre cuando muchas veces el varón que ataca a la mujer lo hace a partir de sentir celos. El varón celoso de nuestro tiempo ya no corre en busca de su rival, al que desafía a través de un duelo; por el contrario, vive atormentado por el goce que le supone a la mujer. "Mientras yo estoy acá hablando, ella seguro está pasándola bomba...", decía un analizante mientras se retorcía en el diván.

Ya no hay duelo, ya no hay hombres. Sólo existen los retornos imaginarios del goce que se supone a las mujeres. Porque, si como decía Lacan en los '70: "La mujer no existe", sólo queda fantasearla. Este es el sueño eterno del mundo contemporáneo. La mujer ya no es objeto, sino sujeto supuesto al goce, y esta hipótesis podría volver inteligible muchos de los actos violentos de esta época.

Por otro lado, en el seminario La relación de objeto (I956-57) Lacan propuso una indicación inquietante al comparar al varón contemporáneo con el caso de Freud conocido como "El pequeño Hans".
La idea también es atractiva. Acaso ¿no se afirma hoy en día que muchos de los hombres son "fóbicos" o, incluso, "histéricos"?

Sin embargo, antes que un tipo clínico, quizá sea más interesante ubicar la posición de niño en que se encuentran los hombres de nuestro tiempo. Esto es algo que también Lacan supo entrever, en el "Discurso de clausura de las Jornadas sobre psicosis en el niño” (I967), cuando se refirió a nuestra época como la del "niño generalizado".

En cierta ocasión, un analizante anunciaba su separación en los siguientes términos: "Soltero de nuevo". La pregunta con que lo interpelamos fue inequívoca: “ ¿Soltero o en adopción?”. Ya no hay hombres, sino niños, en un mundo que sólo ofrece la posibilidad de consumir a falta de experiencia.

Asimismo, de modo ocasional, nos encontramos con sujetos cuya posición de seductores "natos" es particularmente incómoda. La mayoría de la veces se trata de hombres que no pueden dejar de inmiscuirse en diversos deseos con los que se cruzan, al punto de que luego, no pocas veces, terminan quejándose del particular esfuerzo que les requiere estar a la altura de lo que han generado. Como contrapunto, es una queja corriente de las mujeres de nuestra época hablar de una "histeria" masculina, como un modo de referirse a esos hombres que sólo se erotizan preliminarmente, que disfrutan de la seducción y, luego, en el momento de condescender al deseo, desaparecen.

Por cierto, si la cuestión de la seducción no ha despertado demasiado interés en la teoría psicoanalítica, esto puede deberse también a un motivo estructural: por lo general, cuando se interroga la vida amorosa, se intenta esclarecer las condiciones del 
objeto deseado, y no tanto la posición del deseante. Así, por ejemplo, en la primera de las Contribuciones a la psicología del amor, titulada "Sobre un tipo de elección de objeto en el hombre" (I9Io), Freud elucida un tipo particular de interés en el deseo del hombre que requiere la conjunción de diversas "condiciones de amor": a) la condición del "tercero perjudicado", por la cual se elige como objeto de amor a una mujer que no esté "libre", sino a una sobre quien otro hombre puede reclamar "derechos de propiedad"; la mujer que ejerce atracción es aquella cuya castidad puede suponerse en cuestión, o bien a la que puede reputarse una conducta disoluta o infiel; c) estas condiciones, asociadas con una sobrestimación del objeto amado, se repiten varias veces en la historia de la vida amorosa del hombre formando lo que Freud llama "una larga serie", podríamos añadir que se trata de esos hombres que se enamoran siempre "por última vez", es decir, para los cuales la última es siempre la "primera" ("ahora sí estoy enamorado de verdad"); d) en los amantes de este tipo suele exteriorizarse una tendencia particular a querer "rescatar" a las amadas.

De esta presentación de los rasgos de amor de este tipo de elección, la segunda condición de las mencionadas se encuentra vinculada, según Freud, con la cuestión de los celos, sin que quede del todo claro por qué la primera de ellas no lo estaría. En todo caso, podría suponerse que el "derecho de propiedad" cancela el carácter erótico de la mujer para el reclamante; es decir, no es en tanto objeto de deseo que la reclama ese vínculo, podría pensarse aquí, por ejemplo, en la novela El túnel, de E. Sábato, en la que el hecho de que María Iribarne se encuentre casada no es el principal desencadenante de los celos enloquecedores del protagonista, como sí ocurría en el caso de la suposición de un amante (en la segunda condición). Quizá por eso, eventualmente, los hombres pueden bromear y decir, a una mujer casada, "no soy celoso", mientras que enloquecen con la posibilidad de que su amante esté con otro... que no sea su marido.

A propósito de la tercera de las condiciones, cabría apreciar que se vincula directamente con la fascinación del encuentro amoroso, eso que habitualmente llamamos "el flechazo", que ubica inmediatamente al objeto amado en un rango diferencial respecto de las demás objetos.

En relación con la cuarta condición, quizá parezca un poco "desusada" la fantasía de "salvación" de la amada demasiado próximo, tal vez, a ciertos dramas narrativos del siglo xIx, como en la novela Naná de E. Zola; no obstante, podría pensarse en figuras actuales, como la del hombre que se convierte en una suerte de manager de su amada, a la que asiste e intenta orientar en sus proyectos, etc.; en definitiva, de lo que se trata en esta cuarta condición es de la ternura como moción libidinal y de cierto desvalimiento que se le supone al objeto de amor. “¿Qué sería de ella (sin mí)?”, podría parafrasearse esta condición, que no hace más que iluminar en su último tramo el sostén narcisista que la funda y que actualmente se verifica en aquellos hombres que no pueden dejar de “apoyar” (económicamente, emocionalmente, etc.) a sus ex-parejas incluso muchos años después de separados.

De este modo, la seducción se articula con diferentes fantasías que hacen del erotismo una forma variada y singular. 


\section{¿Histeria U HOMOSEXUALIDAD?}

En el seminario La transferencia (I9606I), Lacan se refiere a Sócrates como quien estaría posicionado de manera histérica. La histeria de Sócrates radicaría en su “a-topía”, en la medida en que no es localizable más que de forma excepcional, al responder al discurso del Amo haciendo valer el estatuto de la falta. En la afirmación "Sólo sé que no sé nada" se pone en juego un punto ciego para el saber, que Sócrates, además, nomina con una nueva excepción, relativa, esta vez, a la cuestión del amor.

Si un saldo se desprende de la posición de Sócrates, es que el saber no puede dar cuenta de la diferencia sexuada. Ahora bien, este "imposible saber sobre el sexo" también permite pensar a Sócrates en el lugar del analista -como también lo hace Lacan-. El seminario La transferencia alterna estas dos posibles lecturas de la posición del ateniense. La intervención de Sócrates en el contexto de la interpretación lacaniana de El Banquete, al orientar a Agatón la escena montada por Alcibíades, es una clara ilustración de esta última indicación. A esa referencia vale añadir una segunda, también en clave filosófica, y podría pensarse en Hegel, a quien Lacan diagnostica como "el más sublime de los histéricos".

En principio, cabría preguntarse: ¿por qué Lacan utiliza casos de varones para hablar del paradigma de la histeria? Es evidente su intención de desahuciar el lugar común de relacionar histeria y feminidad -como "cosa de mujeres", de acuerdo con la etimología que vincula el tipo clínico con el útero-; pero no se trata de una provocación, sino que hay también una razón de método: en tanto posición subjetiva, la histeria en la mujer podría estar subtendida por la envidia del pene, pero eso implicaría una interpretación de lo femenino en función de la falta, respecto de la cual el psicoanálisis lacaniano se sitúa en las antípodas. El interés de Lacan, entonces, radica en ubicar que el modelo de la histeria puede ser el varón para establecer, justamente, que la falta fálica no es una condición suficiente. En este punto, el caso de Hegel es emblemático ya que sitúa en primer plano la relación con el saber, en la medida en que éste puede servir al propósito de absolver la disyunción entre el sujeto y lo real. No obstante, aquí se plantea la necesidad de distinguir entre histeria masculina y homosexualidad.

Una de las particularidades de la concepción lacaniana de la homosexualidad radica en diferenciar a esta última de una mera elección de objeto. Ya en el seminario Los escritos técnicos de Frend (I953-54) Lacan hablaba del personaje principal de Proust (en En busca del tiempo perdido) como un homosexual en relación con un objeto heterosexual. La contracara es el caso de la histérica y su hacer de varón (hommesexuel). Ahora bien, ¿de qué modo el histérico varón es también estrictamente "hombre-sexual"?

Por un lado, por su inclusión perfecta en la lógica fálica; pero, en segundo lugar, por la relación con el saber que mencionamos anteriormente. No es casual, entonces, que la primera referencia para hablar de histeria masculina haya sido Sócrates. Desde este punto de vista, lo propio de la histeria -según Lacan- es una relación con el saber, erotizada y basada en la interrogación del amo. No se trata de creer que la histeria se sirve de este último por su deseo, sin más, ya que este aspecto es un rasgo de todas las neurosis, sino que lo particular de la histeria es que ese deseo es 
interrogado por el saber que se le supone. Esto explica también por qué la histerización es una condición del análisis.

Además, en un segundo nivel, la histeria se vincula con la seducción, en la medida en que ésta se establece como coordenada de su fantasma fundamental. La suposición de saber es una versión defensiva respecto del encuentro con un Otro cuyo deseo no tendría objeto, que, como tal, confronta al sujeto con una posición pasiva que declara lo sexual como intrusivo e inasimilable.

Ahora bien, ¿en qué punto la "homosexualidad" intrínseca del sujeto histérico se distingue de la homosexualidad como tipo clínico? En este punto, el libro reciente de Hervé Castanet, Homoanalizantes (2016), permite ubicar que la homosexualidad no es un conjunto homogéneo. A expensas de la posición narcisista, respecto de la división por el sexo puede haber diferentes matices según cómo intervenga el erotismo anal, la pasividad o actividad sexual, la eventual actitud asexuada, los usos de la masturbación, etc. En definitiva, el homosexual es quien rechazó el encuentro con el Otro sexo, en el punto en que podría quedar ubicado más cerca de lo que Lacan llamó "ética del soltero". Teniendo en cuenta que Lacan no se refiere exclusivamente al homosexual masculino, sino al "hommosexual", esto es, aquel que no puede sino ubicarse del lado masculino de las fórmulas de la sexuación. Asimismo, otra dificultad relativa a la homosexualidad radica en que no se ubica en la enseñanza de Lacan una dirección definida. Si en los años '50, en el seminario, desarrolló la idea de una fijación temprana en el segundo tiempo del Edipo (de acuerdo con una relectura del artículo freudiano sobre Leonardo), sobre el fin de esa década re- dactó su escrito "Juventud de Gide" en el que esa elaboración de saber fue criticada clínicamente: André Gide, el que no fue deseado por su madre, el que fue seducido en la pubertad, el homosexual pedófilo, etcetera.

De este modo, si bien puede afirmarse una homosexualidad para el sujeto histérico —en sentido amplio-, la homosexualidad como tal aún permanece como un problema clínico que requiere ser elucidado de manera particular; y, por lo tanto, la interpretación habitual de la homosexualidad latente del histérico no es más que un prejuicio, tanto como lo es que suela afirmarse que los homosexuales tiene una vida promiscua y otras hipotecas heteronormativas en la teoría psicoanalítica.

\section{LO MASCULINO DE LA HISTERIA}

Jacques Lacan afirmaba que el "Don Juan" era una fantasía femenina, en la medida en que este personaje sería una suerte de "varón universal", es decir, ique no existe! ${ }^{1}$ Dicho de otro modo, un hombre que no esté afectado por un deseo que divida a las mujeres es una contradicción. En definitiva, el hombre ideal (el "príncipe azul”) es una fantasía que, quizá, deberíamos pensar más bien como histérica antes que femenina.

Por esta vía, la noción de "envidia del pene” (penisneid) también podría ser matizada como un rasgo propio de la histeria en las mujeres. Esta posición, lo que permite cernirla en un análisis, no radica en que la mujer quiera tener un pene, sino en la actitud de denuncia en que se sitúa para reclamar que ella también debería tenerlo. Dicho de otra forma, esa actitud envidiosa implica una posición de queja, cuyo carácter contradictorio estriba en que se pide algo que, en sentido estricto, 
es dispensable, porque la demanda se sostiene por sí misma. De manera concreta, alcanza con un simple ejercicio para demostrar el carácter histérico de una reivindicación semejante: dar lo que se pide, y que la respuesta sea "no es eso". Sin embargo, a expensas de este rodeo por la posición histérica en las mujeres, interesa ubicar otra cara de esta fantasía de reclamo, esta suerte de afán justiciero, en los varones: lo que podría llamarse la fantasía de "Robin Hood", y que encontramos en muchos sujetos histéricos que, desde una posición heroica, apuntan contra el poder de turno... sin acceder nunca a ese poder. La oposición constante, que sólo puede ser oposición, porque su actitud se sostiene en desconocer el lugar desde el cual ataca a ese Otro que, como en toda fantasía histérica, es el seductor que nos robó traumáticamente el paraíso perdido. El estatus neurótico de esta posición se manifiesta en que también se funda en una contradicción: Robin Hood roba a los ricos, es decir, es un ladrón que roba a otro ladrón y que, por lo tanto, justifica su acto en que no es él quien está detrás de semejante realización (el ladrón ¡es el otro!). Asimismo, el carácter incestuoso de su deseo se expresa en que está destinado a irrealizarse (porque la culpa sería insoportable): es el caso de aquellos que son grandes especialistas en "remarla", pero que con una mano bracean y con la otra se hunden. Esta idea no es para nada novedosa; es lo propio de la actuación histérica, tal como Freud lo entreviera en el caso de la muchacha que con una mano se sube la pollera y con otra se la baja (Cf. Freud, i 908). Para el contexto que aquí concierne, esta circunstancia clínica podría permitir entender muchas de las actuales presentaciones que en la consulta se nombran como "autoboicot" en muchos varones. Sin embargo, una fantasía histérica no alcanza para definir un tipo clínico. Para eso es preciso dar un paso más.

Lo más propio de la histeria es la división subjetiva ante una fantasía de seducción. No obstante, esta coordenada no es idéntica en hombres y mujeres. Para estas, en la medida en que coincide con el Edipo llamado "positivo", la respuesta es más explícita y promueve síntomas "típicos" (Freud, I9I6-I7): la frigidez, el asco, los celos, todas formas de poner en cuestión la actitud receptiva ante un hombre.

Ahora bien, en el caso del varón la cuestión no es tan sencilla, dado que se encuentra imbricada con el Edipo "invertido” y, aquí la cuestión es problemática por el siguiente motivo: no hay respuesta sintomática ante la posición pasiva frente a un hombre. Dicho de otro modo, en esta circunstancia no encontramos los síntomas típicos de la histeria, sino un desarrollo permanente de una actitud defensiva que, por ejemplo, se manifiesta a través de una dedicación constante a tareas (como el trabajo) que, en caso de no estar al altura, se resignifican como pasivización.

A diferencia de la neurosis obsesiva masculina, donde es más frecuente encontrar una sintomatización de la potencia (como impotencia) a través de la comparación, en la histeria de los varones no se trata de las variables habituales de la competencia con otros hombres, sino de una búsqueda permanente de ocupar un lugar fálico. Si no se simboliza el falo, no aparece el síntoma, sino episodios de fuerte angustia (de castración) y, en el lugar en que la obsesión hace una regresión a lo anal, la histeria masculina se desenvuelve más en el campo de una envidia basada en la oralidad. 
Este aspecto se comprueba en una diferencia superficial, pero de cierto interés clínico, que podemos destacar: es más o menos corriente en casos de histeria masculina que sean proclives a prácticas de ejercicio del cuerpo que implican una superación constante en el rendimiento. Aquí el culto de la corporalidad no cuenta como deporte, en el que suele haber rivalidad o técnica (como ocurre, por ejemplo, en el boxeo). Es, por ejemplo, lo que podría pensarse con la práctica actual del crossfit, donde se realiza una práctica de vaciado de goce del cuerpo, en base a su extenuación. Antes que el conteo fálico de la disciplina, aquí se busca esa brecha en que un movimiento apunta al límite en que una flexión no es otra flexión, sino la asíntota entre una y otra. He aquí una forma femenina de suponer un goce o, mejor dicho, una forma histérica de suponer el goce femenino.

Pasemos a un caso. Se trata de un hombre que consulta, luego de un análisis precedente, por un sueño que lo inquietó. Ya de por sí es poco frecuente que alguien consulte por un sueño; no obstante, la reiteración de un sueño que había tenido en su adolescencia (en una época en la que había tenido preguntas relativas a su orientación sexual) le produjo un efecto de angustia: en el sueño, su madre tenía un pene. Si bien se considera heterosexual, está casado y tiene un hijo, aun así busca hacer caso a esa formación de su inconsciente, "porque de la sexualidad nunca se está seguro”.

La pregunta que se le formuló, entonces, fue: “¿Por qué piensa que soñar con una madre con pene implicaría una modificación de su heterosexualidad?". Esta pregunta permaneció como un telón de fondo de varias sesiones, sin respuesta, porque en cierta medida, podría decirse, ya incluía un tipo de respuesta, la del síntoma. Y dado que este hombre ya había transitado anteriormente por la experiencia del análisis, nos autorizamos a una intervención precipitada, en busca de algún efecto asociativo: "Si su madre tiene un pene, eso demuestra que tiene algo mejor de lo que ocuparse... que no es usted". El efecto fue aliviante, y condujo a que este hombre hablara de la relación con su madre, vínculo en el que se recortaba una particular posición que llamó “dependencia”: a pesar de su edad (más de 30 años) todavía conservaba el hábito de pedirle ciertos "favores" a su madre. Le llama la atención de que se trate de tonterías: hacer un trámite, que perfectamente él podría hacer por su cuenta; comprarle medias en un viaje, etc. En este punto se le indicó el carácter sexual que, en nuestro idioma, tiene la palabra "favor". El efecto fue de sorpresa y risa.

En una ocasión posterior, a propósito de la relación con su madre, se define como alguien que hace "ofrendas" a su madre. "Ofrendas para que no se ofenda", respondemos. Esta intervención lo conduce a hablar de lo que implicó la paternidad en su vida. Resume su historia sexual como la de quien pasó de ser un seductor de mujeres a una especie de pollerudo... pero de su mamá. Ha tenido varios problemas con su esposa por el lugar que otorga a su madre en la crianza de su hijo. Advierte que en más de una ocasión le encargó su cuidado en situaciones triviales que podría haber resuelto de otro modo. Se trata, entonces, de un interés -que no logra descifrar muy bien- por el hecho de “apegar” a su hijo con su madre. Su esposa se ha sentido destituida por esta situación, eventualmente le han parecido justi- 
ficados esos celos. Le decimos: "Los celos de su esposa podrían deberse a que usted parece el marido de su madre". "Ah, jel Edipo!", dice este hombre con una sonrisa irónica. "No del todo, porque en este punto no quedaría explicada su pregunta respecto de la homosexualidad. En todo caso, diría que usted ofrece un hijo a su madre, parece un marido, pero para encubrir una relación con su padre: si la madre tiene lo suyo, entonces el padre puede quedar para usted".

Por esta vía, la relación con la madre es el velo de una fantasía de seducción respecto del padre. De este último, el analizante sostiene que se trata de un hombre difícil, del que recuerda una frase en los inicios de la adolescencia: "Tu madre sufre el sexo". Esta afirmación fue elaborada por él en un análisis anterior, en el punto en que pudo advertir el carácter sádico que la relación sexual tendría: un hombre daña a una mujer con su deseo, por eso en su vida erótica él se ofrece en la vía del amor; es generoso, atento, siempre dedicado a satisfacer el goce que le supone a su pareja. Se describe a sí mismo como un gran compañero de las mujeres, el que siempre "pone la oreja" y las contiene. "Un amigo gay”, decimos en chiste.

En otra circunstancia, recuerda una fiesta en la que se encontró con una mujer. Si bien se encuentra casado, tiene diversas relaciones extra-maritales. "No son amantes, yo no tengo amantes, son amigas", dice. Y respecto de la relación con dicha mujer, recuerda el rechazo que sintió por la "calentura", su desprecio por la erección que tuvo al bailar con ella. En este punto, el amor -que incluso puede manifestarse como una celebración de la belleza, como imagen corporal que no se descompleta con un rasgo- sirve como defensa eficaz contra el deseo. En su fantasía, este hombre se defiende de la seducción del padre, a quien queda reservada la posición deseante, y ante esa coyuntura asume la actitud del proveedor: un hijo a la madre, la oreja a las amigas, el goce que le supone a la mujer.

En este punto, si hay un rasgo que se aproxima a un síntoma histérico en este caso es el particular rechazo que le produce su erección, cuando ésta se revela como mera "calentura", y ya no en el marco de una situación seductora. Cuando él ya no es el seductor, es el seducido. Su vida transcurre en una compensación del sentimiento que le produce sentir que podría ser un hombre como los otros, es decir, cortado por la horma del deseo fálico. Es notable que aparezca en el material la cuestión de una pregunta por la homosexualidad; sin embargo, no se trata más que de la pregunta histérica por el ser sexuado que encarna la histeria como tal, es decir, sin recaer en un conflicto respecto de un modo de goce estrictamente homoerótico.

Por último, también el material podría ser leído a partir de la fantasía relativa al goce que se le supone a una mujer, y su elaboración por la vía del saber. Sin embargo, no se trata de una condición suficiente. En el aspecto en que el caso se revela como una forma de histeria masculina remite a un modo de la defensa que, a expensas de un factor conversivo, podría ser pensado como una forma mínima del asco (ese rechazo).

\section{Conclusiones}

Los tres apartados precedentes podrían ser pensados de acuerdo con la siguiente secuencia argumentativa: en primer lugar, es preciso diferenciar la histeria como tipo 
clínico de la posición seductora (o "histerizada" en sentido general); en segundo lugar, es preciso no confundir la histeria masculina con una homosexualidad latente, según una interpretación habitual en ciertos psicoanalistas, ya que la homosexualidad dista de ser algo homogéneo y, al mismo tiempo, no se trata de un tipo clínico. Por esta vía es preciso continuar con trabajos que incorporen ciertos matices de género en la clínica psicoanalítica para deshacer prejuicios arraigados.

Por último, al situar la histeria masculina en relación con la defensa respecto de un fantasma de seducción, queda una pregunta: ¿cómo pensar un tipo clínico sin basarlo en un mecanismo de formación de síntoma? O, al menos, sin considerar síntomas "típicos" como los que se encuentran en la histeria femenina. En este sentido, nuestro trabajo parece pisarse la cola, ya que propone un supuesto $y$, en el curso de su argumento, demuestra su carácter problemático. Sin embargo, éste ha sido el objetivo principal de nuestro artículo, llegar a este problema clínico que -creemoses el que implica sostener la categoría de histeria masculina como una de las formas que adopta la neurosis.

\section{NOTAS AMPLIATORIAS}

1 Don Juan, es decir, aquel que sería capaz de ver la singularidad de cada mujer; o, dicho de otro modo, ese hombre que podría apreciar a cada mujer como única, para el cual sólo existirían las mujeres y nunca buscaría en una los rastros de otra. No obstante, este hombre no existe. Y, según Lacan, habría que entreverlo como un fantasma femenino: "Si el fantasma de Don Juan es un fantasma femenino, es porque responde al anhelo de la mujer de una imagen que desempeñe su función, función fantasmática -que haya uno, un hombre, que lo tenga- lo cual, en vista de la experiencia, es un desconocimiento de la realidad -todavía más, que lo tenga siempre, que no pueda perderlo. Lo que implica precisamente la posición de Don Juan en el fantasma es que ninguna mujer puede arrebatárselo, he aquí lo esencial. Es lo que él tiene en común con la mujer, a quien, por supuesto, no puede serle arrebatado porque no lo tiene" (LACAN, I962-63, p.219). La mujer imagina que podría haber un hombre que no estuviese atravesado por la castración. Sería un hombre, entonces, al que nada le faltaría... como a la mujer -he aquí por qué Lacan dice que se trata de un fantasma femenino, aunque sería más correcto decir que se trata de un fantasma neurótico que imagina en el hombre un goce simétrico al de la mujer. Podría pensarse, por ejemplo, en el caso del padre de la histérica, cuya castración es objetada por el síntoma, en la medida en que este último le está ofrendado. El síntoma histérico es un monumento a la idealización del padre, a la potencia del padre (aunque más no sea para demostrar la inscripción de su impotencia, como lo demuestra el caso Dora; cf. Freud, I905, p.42), el primer seductor que admitiría la estructura. Cabe recordar que, ya en el comienzo de su práctica, Freud se encontró con la cuestión de la queja respecto de la seducción en la histeria, al punto de apreciar que se trataba de una fantasía y no de un hecho 
efectivamente vivido -o bien, independientemente de lo acontecido, lo que importaba era la posición pasiva asumida por el sujeto en la fantasía. Ese Otro seductor no es el partenaire al que muchas veces la histérica ataca furiosamente (y que, eventualmente, suele representar el lugar de competencia fálica con algún hermano), ni el seductor efectivo que puede piropearla en la calle (y al que puede responder con diversas actitudes, desde la indiferencia hasta la sonrisa), sino que se trata de una función de reserva fálica, que sostiene un ideal de existencia de "uno que no" (no afectado por la castración). Por eso, incluso podría pensarse que el mito freudiano del padre de la Horda -elaborado en Tótem y tabú (I9I3)- es una suerte de fantasma femenino, que supone que habría un padre que gozaría de todas las mujeres o, mejor dicho, que podría gozar de todas la mujeres sin verse afectado por la detumescencia, por el carácter discontinuo del goce fálico, asociado a la insatisfacción. Ese lugar que la histeria suele reservar al padre, en el amor, puede ocasionalmente encarnarlo el partenaire en la figura de esos maridos que requieren todo tipo de atenciones; que, a primera vista, son todo lo contrario a un seductor, pero sostienen esta función fantasmática de la excepción. De este modo, puede verse cómo el donjuanismo no está asociado a la delicadeza o al mero coqueteo de que puede hacer gala el hombre. En todo caso, estas actitudes remiten al pavoneo fálico con el que un hombre puede "vestirse" -su relativa impostura- para demostrar su interés por una mujer. Pero el caso del Don Juan, como fantasía femenina, remite a ese punto en que ese hombre -que se supone que existe- no estaría interesado por ninguna. $\mathrm{Al}$ igual que al padre de la Horda, le corresponderían todas, pero este no sería sino un modo de indicar que desea a ninguna. En este punto, cabría trazar una distinción entre Don Juan y el padre de la Horda: "Casi parece un camelo subrayar la relación de Don Juan con la imagen del padre en tanto que no castrado. Quizás lo sea señalar que se trata de una pura imagen femenina" (LACAN, I962-63, p.209). El argumento de Lacan no parece concluyente. ¿Por qué el hecho de que se trate de un fantasma femenino debería llevar a distinguirlo de la función del padre de la Horda? En principio, porque este último es una función estructural de todo fantasma neurótico. En todo caso, cabría pensar que el Don Juan es la versión histérica del padre de la Horda. Así parece entreverlo Lacan en el seminario 10 cuando describe la práctica mítica del derecho de pernada y otros ritos de desfloración. $\mathrm{Cu}$ riosamente, quien se encargaba de estos actos era el sacerdote de una sociedad, a un tiempo representante de la función paterna, pero también de quien se esperaría que no sea un galán, sino que haga su trabajo. Por eso, la función del donjuanismo no nombra lo que habitualmente llamamos un "Don Juan" -el mujeriego-, sino una condición estructural: "La huella sensible de lo que les planteo acerca de Don Juan es que la compleja relación del hombre con su objeto está borrada para él, pero a costa de aceptar su impostura radical. El prestigio de Don Juan está ligado a la aceptación de dicha impostura" (LACAN, I962-63, p.209). Dado que para él está borrada la relación con el objeto, por lo tanto, Don Juan no es un hombre deseante. De este modo, cumple asimismo -como todo fantasmauna función defensiva: "Hay que decirlo, no es un personaje angustiante para la mujer. Cuando sucede que una mujer siente que es verdaderamente el objeto en el centro de un deseo, pues bien, créanme, de esto es de lo que en verdad huye” (LACAN, I962-63, p.210). En definitiva, el fantasma de Don Juan es una forma de defensa contra el interés (y el deseo) que un hombre podría manifestar por una mujer. Una deriva de este ponerse a resguardo se da a través de la idealización del hombre, al cual se le supone que podría tener a todas las mujeres, como un modo de indeterminar el carácter singular del deseo. 
Otra deriva podría estar en un fantasma de celos y, en este caso sí, en la suposición de que el hombre es un mujeriego, como una manera de salir del "centro". Ambos aspectos podrían resumirse en la idea de que la habitual acusación de donjuanismo que las mujeres reprochan a los hombres aúna un componente celotípico tanto como cierta idealización. Por esta vía, es curioso advertir que la atribución de un más allá de la castración termina siendo un modo de rechazar una condición deseante; o bien, es un modo de volver a notar que, en psicoanálisis, la castración es constitutiva del deseo.

\section{REFERENCIAS BIBLIOGRÁFICAS}

Bleichmar, S. (2006). Paradojas de la sexualidad masculina, Buenos Aires, Paidós.

Castanet, H. (2016). Homoanalizantes, Buenos Aires, Grama.

Freud, S. (1905). "Fragmento de análisis de un caso de Histeria (Dora)", en Obras Completas (Vol 12), Buenos Aires, Amorrortu.

(I9I0). "Contribuciones a la psicología del amor”, en Obras Completas (Vol 2), Buenos Aires, Amorrortu. (1916-I7). Conferencias de introducción al psicoanálisis, en Obras Completas (Vol 16), Buenos Aires, Amorrortu.

LACAN, J. (1953-54). El seminario 1: Los escritos técnicos de Freud, Buenos Aires, Paidós.

(1956-57). El seminario 4: La re-

lación de objeto, Buenos Aires, Paidós.
(1960-6I). El seminario 8: La transferencia, Buenos Aires, Paidós. (1962-63). El seminario 10: $\mathrm{La}$ angustia, Buenos Aires, Paidós. (1967) "Discurso de clausura de las Jornadas sobre psicosis en el niño", inédito.

(I973) "Psicoanálisis, Radiofonia $y$ Television”, Buenos Aires, Anagrama.

\section{RESEÑA CURRICULAR DEL AUTOR}

Luciano Lutereau, es psicoanalista, Doctor en Filosofía y Doctor en Psicología, UBA. Magister en Psicoanálisis y Especialista en Psicología Clínica por la misma Universidad, donde trabaja como docente e investigador. Dirige la Licenciatura en Filosofia en UCES. Autor de varios libros, entre ellos: "La experiencia analítica. De la técnica a la ética" (Buenos Aires, Letra viva, 20I7). 